\title{
ASKETOGENYS ACUBECCA, A NEW GENUS AND SPECIES OF DACETINE ANTS FROM MALAYA (HYMENOPTERA: FORMICIDAE)
}

\author{
By William L. BRown, JR. ${ }^{1}$
}

\begin{abstract}
Asketogenys new genus
Worker: With the general characters of Smithistruma, but differing in the form and dentition of the mandibles: each shaft moderately elongate and gently arcuate, subporrect, with a submedian cluster of 3 stout teeth; distal to this a large diastema containing a single small, isolated subapical tooth; downturned apex with a close series of minute teeth and denticles; apical half of mandible also with 3 conspicuous paddle-shaped hairs curving dorsomesad from the dorsal surface. Free clypeal margin with a slender anteromedian spine. Head narrow, subcuneiform, with shallow posterior excision. Middle of vertex impressed. Antennae slender, 6-merous in the usual strumigenite proportions. Eyes small.

Trunk slender, with conspicuous, raised lamellar dorsolateral margins throughout, culminating in acute propodeal spines. Petiolar node with a median lamellar fimbria on its anterior face; both nodes with voluminous spongiform appendages. Legs long and slender.

Sculpture predominantly reticulate-punctulate; gaster smooth, its tergum basally costulate in the usual manner. Specialized hairs fairly numerous, long, flagelliform. Color ferruginous. Male and queen unknown.

Type species, by present designation:
\end{abstract}

\section{Asketogenys acubecca new species}

(Figs. I, 2)

Holotype worker: Total length (TL) 2.7, head length (HL) 0.65 , head width (HW) 0.40 (cephalic index 6r), mandible length (ML) 0.195 (mandibulo-cephalic index, or MI, 30), trunk length (WL) $0.7 \mathrm{I}$, scape $\mathrm{L} 0.45$, greatest diameter of eye 0.03 , $\mathrm{L}$ hind femur $0.57, \mathrm{~L}$ hind tibia $0.4 \mathrm{I}, \mathrm{L}$ hind tarsus including claws 0.85 , $\mathrm{L}$ hind metatarsus $0.45 \mathrm{~mm}$. Petiolar node (without appendages) $0.12 \mathrm{~mm}$ wide; disc of postpetiole $0.10 \mathrm{~mm}$ long, $0.22 \mathrm{~mm}$ wide.

\footnotetext{
${ }^{1}$ Department of Entomology, New York State College of Agriculture and Life Sciences at Cornell University, Ithaca, New York 14850, USA. Research and publication aided by grants nos. 5574X and 31662X from the National Science Foundation.
}

Manuscript received by the editor, March 24, 1972. 


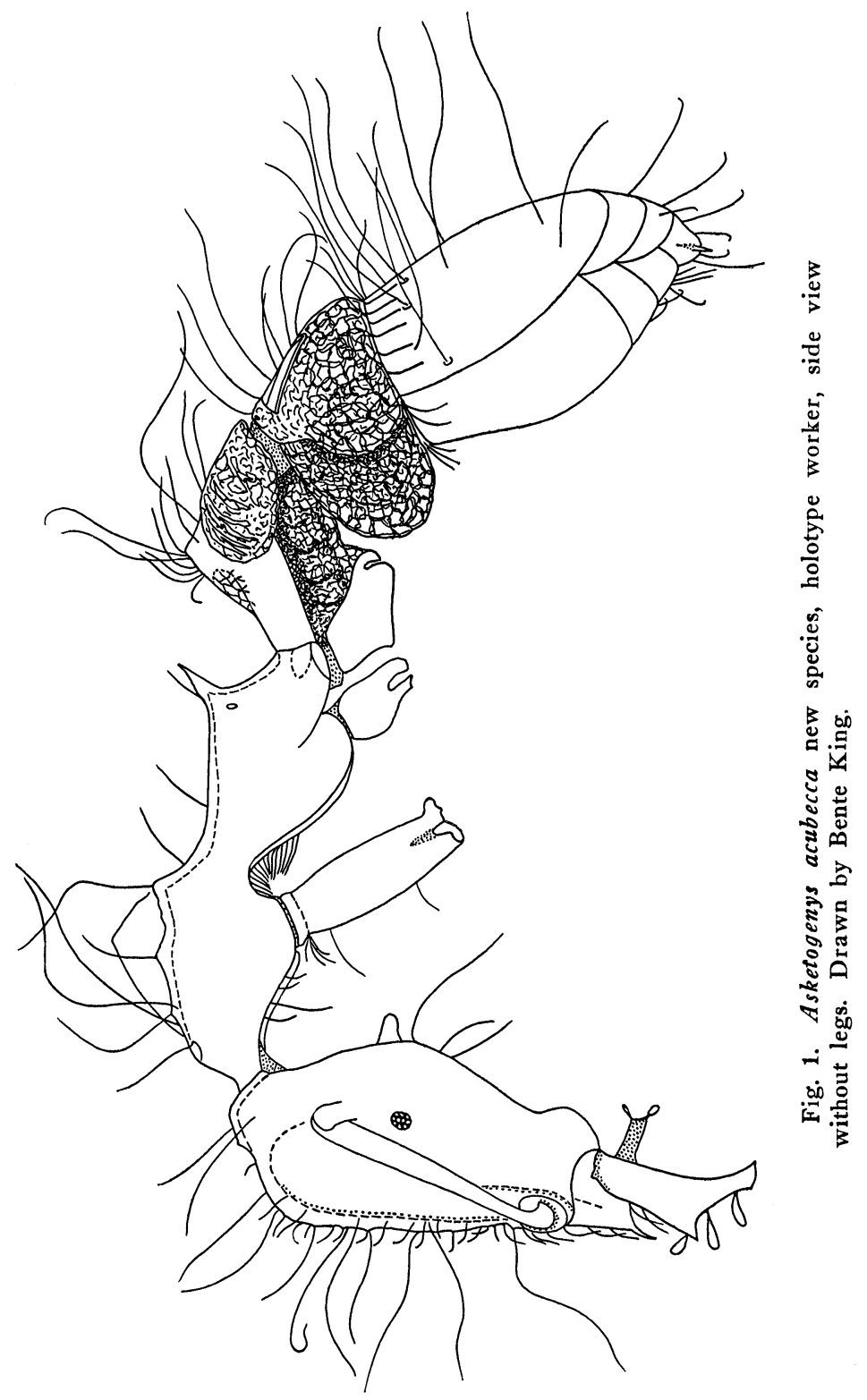




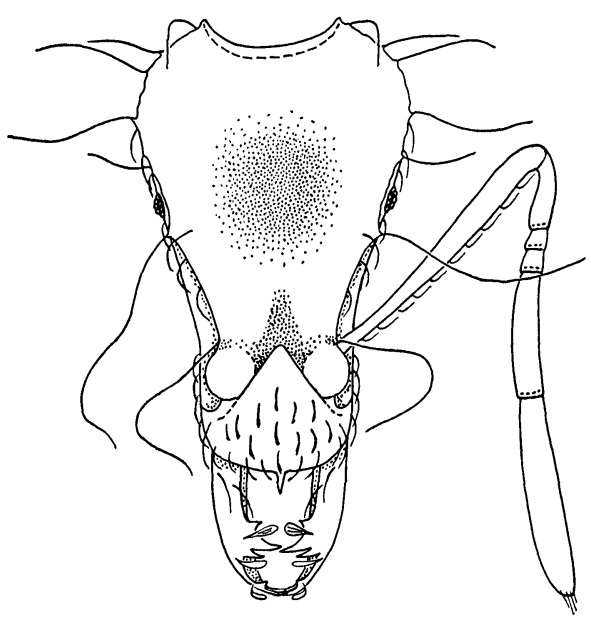

Fig. 2. Asketogenys acubecca new species, holotype worker, full-face (dorsal) view of head, only fringing pilosity shown for upper part. Drawn by Bente King.

Description will be limited to details not evident from the generic characterization and the figures. The stippled area of Fig. 2 indicates the position of the impressed area on the vertex. Promesonotum flat, with upturned lamellar margins as shown; the margins become less distinct posteriad on the propodeal dorsum (which is gently convex from side to side), and finally almost disappear just in front of the propodeal teeth. Posterodorsal face of petiolar node nearly square; disc of postpetiolar node transversely elliptical.

Details of the mandibular bases are unknown; presumably an erect basal lamella is present beneath the clypeus. The labrum is of the Smithistruma pattern, with paired long-subconical lobes bearing a pair of short spatulate trigger hairs at the apex. The mandibular apex, difficult to see in this case at full closure, consists of a very short border, nearly perpendicular to the main axis of the shaft, onto which are crowded a very small but acute dorsal (proximal) tooth (which crosses its opposite number at full closure), about six minute denticles, and a very small ventral (apical) tooth. This apical arrangement reminds one of some Epitritus species.

Head, trunk and coxae densely reticulate-punctulate throughout, the individual punctulae moderately coarse and with shining bottoms, but the general surface prevailingly opaque, except for a weakly shining area on each posterior side of trunk. Clypeus, dorsal and 
lateral surfaces of mandibles, antennae, cervix, legs and petiole very densely and finely punctulate, opaque except for lower sides of petiolar peduncle and posterodorsal face of petiolar node, which are weakly shining. Disc of postpetiole and all of gaster smooth and shining. Costulae at base of gaster fine, numerous, extending about $\mathrm{I} / 4$ the length of the basal segment.

Specialized erect hairs long and fine, up to about $0.4 \mathrm{~mm}$ long, generally distributed over both surfaces of head behind clypeus, dorsa of trunk and both nodes, both surfaces of gaster, and on legs as far out as metatarsi. Paddle-shaped hairs of mandibles as shown in the figures. Ground pilosity of head consisting of fine, curved, short hairs - longer and standing more away from the surface on vertex, slightly broadened and appressed to subappressed on clypeus. Legs, underside of head, pronotal cervix and antennae with fine appressed and subappressed short hairs. Gastric apex with some short fine erect hairs.

Color medium ferruginous, gaster a shade darker, more brownish; antennae more yellowish.

Holotype (unique) from Trolac Forest Reserve, Perak, Malaysia, 6 August 1967, leaf litter berlesate from second growth rain forest (R. H. Crozier leg.), deposited in the Museum of Comparative Zoology, Harvard University, Cambridge, Massachusetts.

This odd species is related most closely to Smithistruma, but the unique mandibles and marginate trunk take it out of that genus. The anterior clypeal tooth is unknown in Smithistruma, but a similar, undoubtedly convergent structure is known in a species of Gymnomyrmex (dentinasis) from southern Brasil. Gymnomyrmex, though very different from the "average" Smithistruma, may be connected to the latter by a chain of intergradient species. In fact, the material now available indicates that the time for amalgamation of several of the short-mandibulate strumigenite genera is approaching. Unless new intergradient species are found in the future to connect Asketogenys acubecca to some other short-mandibulate strumigenite genus, it is distinct enough to survive any reasonable foreseeable revision as a genus apart. 

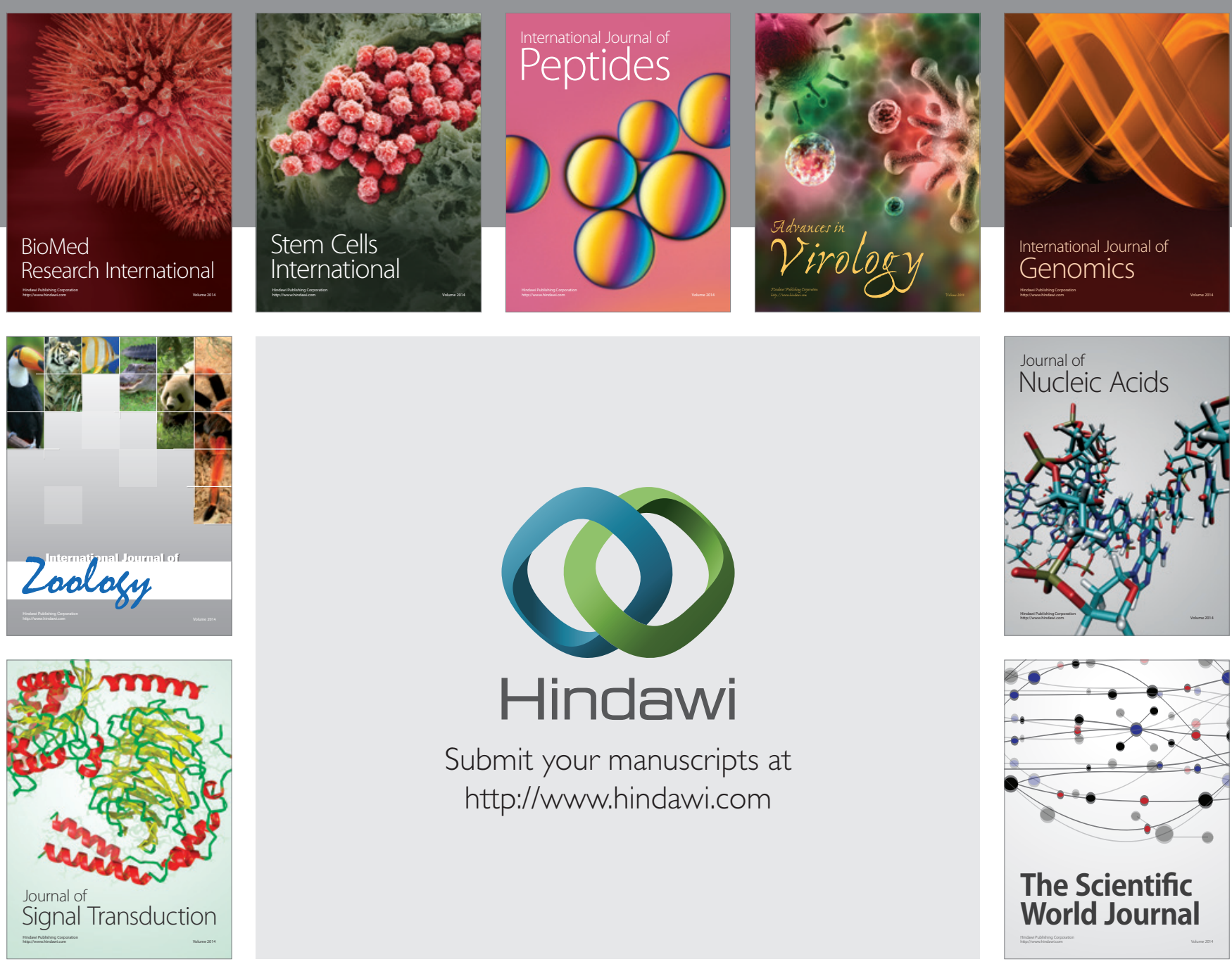

Submit your manuscripts at

http://www.hindawi.com
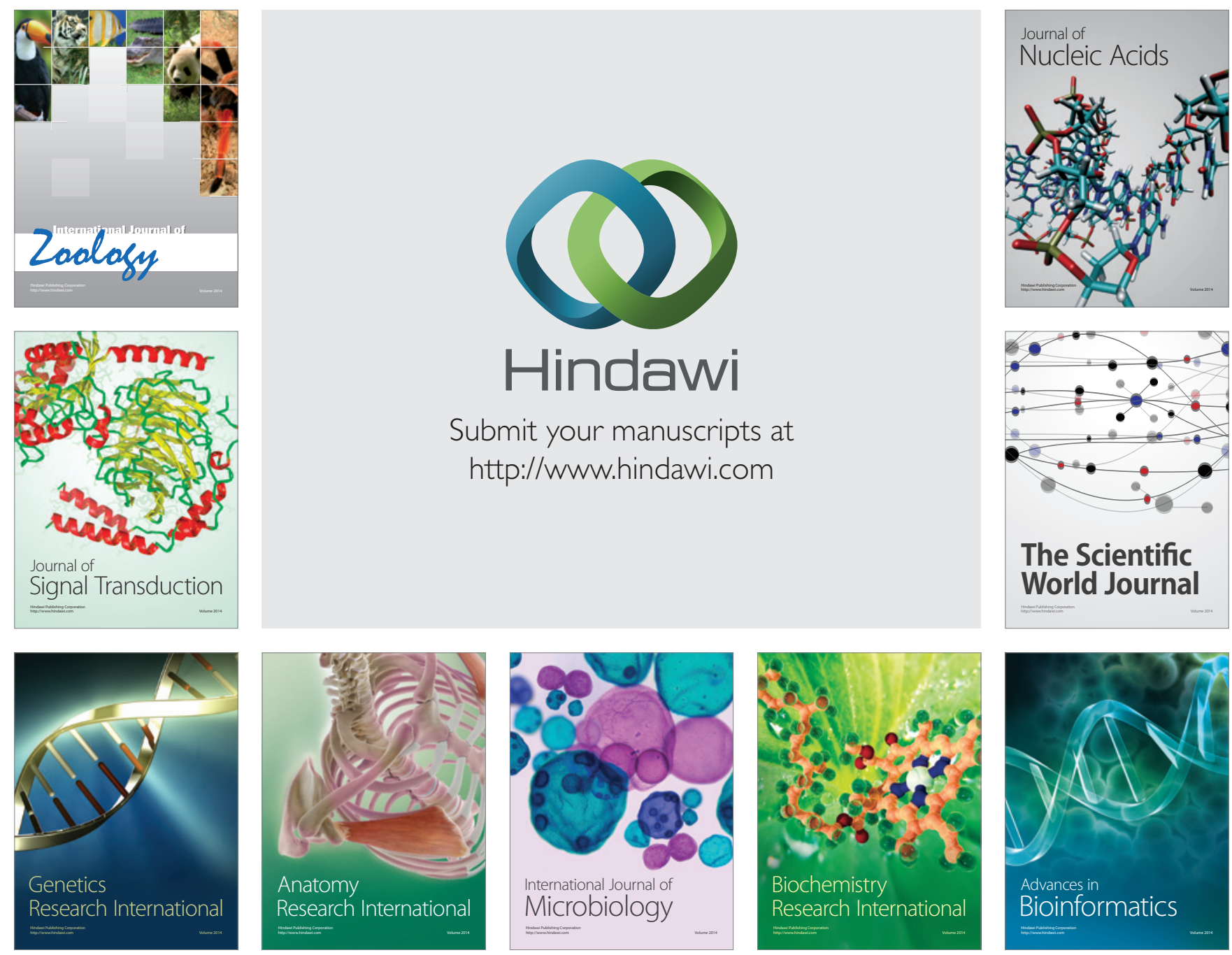

The Scientific World Journal
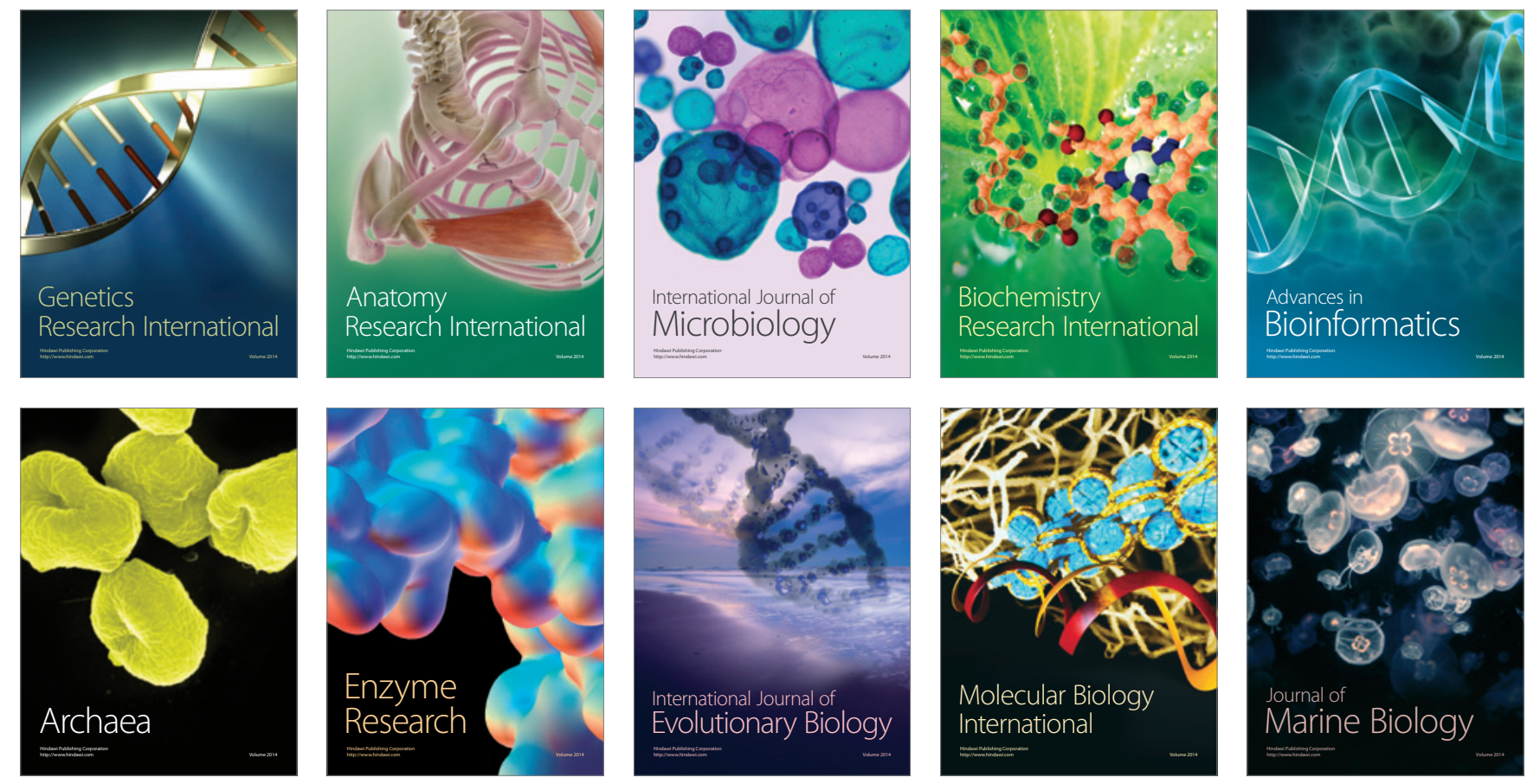